\title{
AdapterDrop: On the Efficiency of Adapters in Transformers
}

\author{
Andreas Rücklé* and Gregor Geigle and Max Glockner, \\ Tilman Beck and Jonas Pfeiffer and Nils Reimers and Iryna Gurevych \\ Ubiquitous Knowledge Processing Lab (UKP) \\ Department of Computer Science, Technische Universität Darmstadt \\ www.ukp.tu-darmstadt.de
}

\begin{abstract}
Transformer models are expensive to fine-tune, slow for inference, and have large storage requirements. Recent approaches tackle these shortcomings by training smaller models, dynamically reducing the model size, and by training light-weight adapters. In this paper, we propose AdapterDrop, removing adapters from lower transformer layers during training and inference, which incorporates concepts from all three directions. We show that AdapterDrop can dynamically reduce the computational overhead when performing inference over multiple tasks simultaneously, with minimal decrease in task performances. We further prune adapters from AdapterFusion, which improves the inference efficiency while maintaining the task performances entirely.
\end{abstract}

\section{Introduction}

While transfer learning has become the go-to method for solving NLP tasks (Pan and Yang, 2010; Torrey and Shavlik, 2010; Ruder, 2019; Howard and Ruder, 2018; Peters et al., 2018), transformerbased models are notoriously deep requiring millions or even billions of parameters (Radford et al., 2018; Devlin et al., 2019; Radford et al., 2019; Liu et al., 2019; Brown et al., 2020). This results in slow inference and large storage requirements.

At least three independent lines of research have recently evolved to tackle these shortcomings. (1) Smaller and faster models that are either distilled or trained from scratch (Sanh et al., 2019; Sun et al., 2020; Bai et al., 2021; Wang et al., 2020). (2) Robustly trained transformers in which the model depth can be reduced at run-time, thereby decreasing inference time dynamically (Fan et al., 2020; Elbayad et al., 2020; Xin et al., 2020; Hou et al., 2020). (3) Adapters, which, instead of fully fine-tuning the model, only train a newly introduced set of weights at every layer, thereby sharing

${ }^{*}$ Work done prior to joining Amazon. the majority of parameters between tasks (Houlsby et al., 2019; Bapna and Firat, 2019; Pfeiffer et al., 2020a). Adapters have been shown to work well for machine translation (Bapna and Firat, 2019), cross-lingual transfer (Pfeiffer et al., 2020b, 2021b; Üstün et al., 2020; Vidoni et al., 2020; Ansell et al., 2021), community QA (Rücklé et al., 2020), and task composition for transfer learning (Stickland and Murray, 2019; Pfeiffer et al., 2021a; Lauscher et al., 2020; Wang et al., 2021; Poth et al., 2021). Despite their recent popularity, the computational efficiency of adapters has not been explored beyond parameter efficiency.

We close this gap and establish the computational efficiency of two adapter architectures at training and inference time. We investigate different strategies to further improve the efficiency of adapter-based models by incorporating ideas from all three directions mentioned above. Our strategies rely on dropping out adapters from transformers, at training and inference time, resulting in models that are dynamically adjustable regarding the available computational resources. Our approaches are agnostic to the pre-trained transformer model (e.g., base, large), which makes them broadly applicable.

\section{Contributions:}

1. We are the first to establish the computational efficiency of adapters compared to full fine-tuning. We show that the training steps of adapters can be up to $60 \%$ faster than full model fine-tuning with common hyperparameter choices, while being 4-6\% slower at inference. Hence, adapters are a suitable choice for researchers interested in achieving faster training times, or when requiring extensive hyperparameter tuning.

2. We propose AdapterDrop, the efficient and dynamic removal of adapters with minimal impact on the task performances. We show that dropping adapters from lower transformer layers considerably improves the inference speed in 


\begin{tabular}{llrrrr}
\hline Setting & Adapter & \multicolumn{4}{c}{ Relative speed (for Seq.Len./Batch) } \\
\cline { 3 - 6 } & & $128 / 16$ & $128 / 32$ & $512 / 16$ & $512 / 32$ \\
\hline Training & Houlsby & 1.48 & 1.53 & 1.36 & 1.33 \\
& Pfeiffer & 1.57 & 1.60 & 1.41 & 1.37 \\
Inference & Houlsby & 0.94 & 0.94 & 0.96 & 0.96 \\
& Pfeiffer & 0.95 & 0.95 & 0.96 & 0.96 \\
\hline
\end{tabular}

Table 1: Relative speed of adapters compared to fully fine-tuned models. For example, 1.6 for training with the Pfeiffer adapter means that we can perform 1.6 training steps with this adapter in the time of one training step with full model fine-tuning.

multi-task settings. For example, with adapters dropped from the first five layers, AdapterDrop is $39 \%$ faster when performing inference on 8 tasks simultaneously. This can be beneficial for researchers working on models that need to make multiple predictions on each input.

3. We prune adapters from adapter compositions in AdapterFusion (Pfeiffer et al., 2021a) and retain only the most important adapters after transfer learning, resulting in faster inference while maintaining the task performances entirely. This is suitable for settings with little labeled training data, where AdapterFusion can achieve ample improvements over standard single task models.

\section{Efficiency of Adapters}

We first establish the computational efficiency of adapters without AdapterDrop. As illustrated in Figure 1, significant differences exist in the forward and backward pass when fine-tuning adapters compared to fully fine-tuning the model. In the forward pass, adapters add complexity with the additional components; however, it is not necessary to backpropagate through the entire model during the backward pass. We compare the training and inference speed of full model fine-tuning against the adapter architectures of Houlsby et al. (2019) and Pfeiffer et al. (2021a) (depicted in Figure 1) using the AdapterHub.ml framework (Pfeiffer et al., 2020a). We conduct our measurements with the transformer configuration of BERT base and verify them with different GPUs. ${ }^{1}$

We provide measurements corresponding to common experiment configurations in Table 1.

Training. Adapters can be considerably faster compared to full model fine-tuning- $60 \%$ faster

\footnotetext{
${ }^{1}$ We experiment with newer and older GPUs, Nvidia V100 and Titan X, respectively. See Appendix A.1 for details.
}

in some configurations. The two adapter architectures differ only marginally in terms of training efficiency: due to its simpler architecture, training steps of the Pfeiffer adapters are slightly faster. The magnitude of the differences depends on the input size; the available CUDA cores are the primary bottleneck. ${ }^{2}$ We do not observe any particular differences between adapters and full fine-tuning regarding the training convergence. ${ }^{3}$

The training speedup can be explained by the decreased overhead of gradient computation. Most of the parameters are frozen when using adapters and it is not necessary to backpropagate through the first components (see Figure 1).

Inference. The two adapter architectures are 94$96 \%$ as fast as fully fine-tuned models, which varies depending on the input size. This can have a considerable impact when deployed at scale.

\section{AdapterDrop}

We have established that adapters are more efficient in terms of training time, however, there is a perpetuate need for sustainable and efficient models (Strubell et al., 2019). Backpropagating through as few layers as possible would further improve the efficiency of training adapters. The efficiency for inference can be improved by sharing representations at lower transformer layers when simultaneously performing inference for multiple tasks-in other words, when performing multiple independent classifications on the same input. We establish this in Table 2, finding that models are up to $8.4 \%$ faster with every shared layer (16 tasks).

Motivated by these observations, we propose AdapterDrop: Dynamically removing adapters from lower transformer layers (depicted in Figure 1). AdapterDrop is similar to dropping out entire transformer layers (Fan et al., 2020), however, specialized to adapter settings-where lower layers often have a small impact on the task performances (Houlsby et al., 2019).

We study two training methods for AdapterDrop: (1) Specialized AdapterDrop: Removing adapters from the first $n$ transformer layers, where $n$ is fixed during training. This yields separate models for each possible $n$. (2) Robust AdapterDrop: Drawing the integer $n$ randomly from $[0,11]$ for each

\footnotetext{
${ }^{2}$ We include detailed plots in Appendix G.1.

${ }^{3}$ We also pre-train adapters with masked language modeling, finding that this does not yield better results (Appendix B).
} 

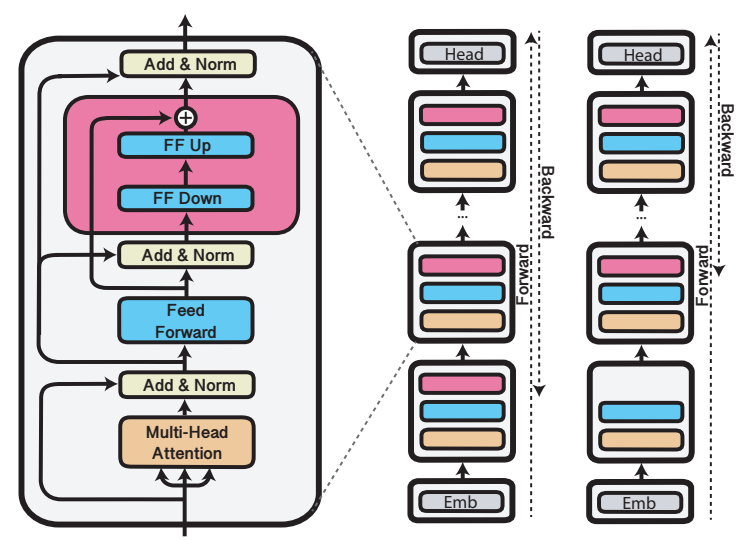

Figure 1: Standard adapter fine-tuning vs. AdapterDrop fine-tuning. The left model includes adapters at every layer whereas the right model has adapters dropped at the first layer. The arrows to the right of each model indicate the information flow for the Forward and Backward pass through the model.

\begin{tabular}{lrrrr}
\hline Simultaneous Tasks & 2 & 4 & 8 & 16 \\
\hline Speedup (each layer) & $4.3 \%$ & $6.6 \%$ & $7.8 \%$ & $8.4 \%$ \\
\hline
\end{tabular}

Table 2: Speedup for each shared transformer layer when performing inference for multiple tasks simultaneously (details are given in Appendix G.2)

training batch. ${ }^{4}$ This yields one robust model that is applicable to a varying number of dropped layers. We study the effectiveness of AdapterDrop on the devsets of the GLUE benchmark (Wang et al., 2018) using RoBERTa base (Liu et al., 2019). ${ }^{5}$

Figure 2 shows that specialized AdapterDrop maintains good results even with several dropped layers. With the first five layers dropped, specialized AdapterDrop maintains $97.1 \%$ of the original performance (averaged over all eight GLUE tasks; see Table 8). Moreover, robust AdapterDrop achieves comparable results, and with five layers dropped it maintains $95.4 \%$ of the original performance (on avg). The advantage of robust over specialized AdapterDrop is that the robust variant can be dynamically scaled. Based on current available computational resources, robust AdapterDrop can (de)activate layers with the same set of parameters, whereas specialized AdapterDrop needs to be trained for every setting explicitly.

The efficiency gains can be large. When performing inference for multiple tasks simultaneously, we measure inference speedups of $21-42 \%$ with five

\footnotetext{
${ }^{4}$ We also explored dropping adapters from randomly chosen layers (instead of early layers). This generally performs worse and it requires selecting a suitable dropout rate.

${ }^{5}$ The detailed setup is listed in Appendix A.2.
}

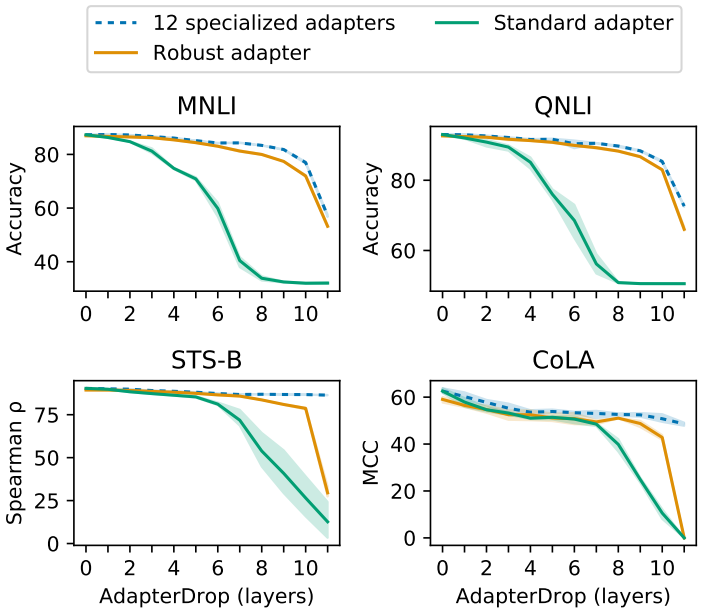

Figure 2: Task performances in relation to dropped layers during evaluation (Figure 13 shows all tasks). 'Standard adapter' is trained with no dropped layers.

dropped layers-depending on the number of simultaneous tasks (Table 2). ${ }^{6}$ Training of our robust adapters is also more efficient, which increases the speed of training steps by $26 \%{ }^{7}$

\section{Efficiency of AdapterFusion}

AdapterFusion (Pfeiffer et al., 2021a) leverages the knowledge of several adapters from different tasks and learns an optimal combination of the adapters' output representations for a single target task (see Figure 3). AdapterFusion (AF) is particularly useful for small training sets where learning adequate models is difficult. Despite its effectiveness, AF is computationally expensive because all included adapters are passed through sequentially. ${ }^{8}$

Table 3 shows that the differences can be substantial for both training and inference. For instance, compared to a fully fine-tuned model, AF with eight adapters is around $47 \%$ slower at training time and $62 \%$ slower at inference. ${ }^{9}$

\section{AdapterDrop for AdapterFusion}

There exists considerable potential for improving the efficiency of AF, especially at inference time. We address this with two variants of AdapterDrop

\footnotetext{
${ }^{6}$ For more details see Appendix G.2

${ }^{7}$ Every dropped adapter improves the speed of training steps by $4.7 \%$ and we drop on average 5.5 adapters when training robust adapter models (more hyperparameter settings and details are given in Appendix G.2).

${ }^{8}$ We also test AF with parallel operations and found no efficiency gains (see Appendix $\mathrm{H}$ ).

${ }^{9}$ All with Pfeiffer adapter and depending on the input size. We provide more measurements in Appendix G.3.
} 


\begin{tabular}{rrrrrr}
\hline & \multicolumn{2}{c}{ AF vs. Full FT } & & \multicolumn{2}{c}{ AF vs. Adapter } \\
\cline { 2 - 3 } \cline { 5 - 6 } Adapters & Training & Inference & & Training & Inference \\
\hline $\mathbf{2}$ & 0.92 & 0.64 & & 0.57 & 0.68 \\
$\mathbf{8}$ & 0.53 & 0.38 & & 0.33 & 0.40 \\
$\mathbf{1 6}$ & 0.33 & 0.24 & & 0.21 & 0.26 \\
\hline
\end{tabular}

Table 3: Relative speed of AdapterFusion (with 2/8/16 adapters) compared to a fully fine-tuned model and compared to a single-task adapter (right). Measured with a batch size of 32, and a sequence length of 128 .

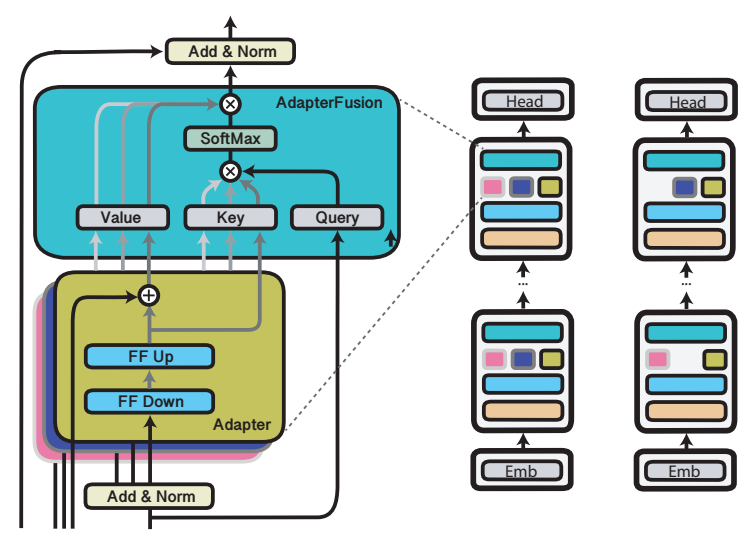

Figure 3: Standard AdapterFusion vs. AdapterFusion pruning, each with 3 adapters initially. The left model includes all adapters at every layer whereas the right model has one adapter pruned at every layer.

for AF by (1) removing entire AF layers; (2) pruning the least important adapters from AF models.

\subsection{Removing AdapterFusion Layers}

We fuse the adapters from all eight GLUE tasks and observe the largest gains of AF on RTE and CoLA. We additionally train robust AF models with the same procedure as in $\S 3$. We investigate from how many lower layers we can remove $\mathrm{AF}$ at test time while still outperforming the corresponding singletask adapter (without AdapterDrop).

Figure 4 shows that AF performs better than the

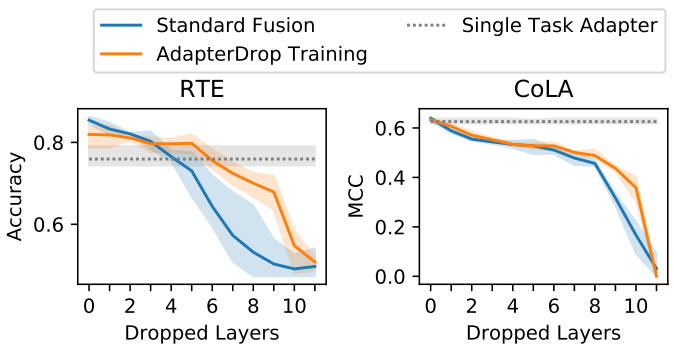

Figure 4: Comparison of AdapterFusion with (orange) and without (blue) AdapterDrop training during inference when omitting early AF layers.

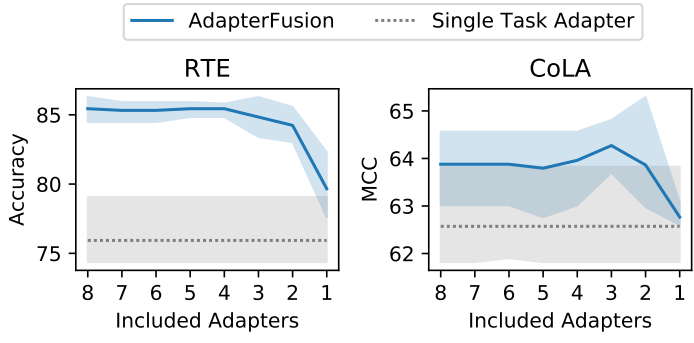

Figure 5: Task performance of AdapterFusion Pruning. $\mathrm{AF}$ is trained with eight adapters, and we gradually remove the least important from the model.

single-task adapter on RTE until removing AF from the first five layers. This improves the inference efficiency by $26 \% .{ }^{10}$ On CoLA, we observe a different trend. Removing AF from the first layer results in more noticeable performance decreases, achieving lower task performances than the single-task adapter. This is in line with recent work showing that some linguistic tasks heavily rely on information from the first layers (Vulić et al., 2020). We deliberately highlight that AdapterDrop might not be suitable for all tasks. However, Figure 13 shows that CoLA represents the most extreme case. Nevertheless, our results suggest that researchers need to be cautious when removing AdapterFusion layers as there may exist a considerable performance/efficiency tradeoff.

\subsection{AdapterFusion Pruning}

The inference efficiency of AF largely depends on the number of fused adapters, see Table 3. We can, therefore, achieve efficiency improvements by pruning adapters from the trained AF models (depicted in Figure 3). Our hypothesis is that we can safely remove adapters if they are not usually activated by AF, which means that they do not contribute much to the output representations. In each fusion layer, we record the average adapter activations - their relative importance-using all instances of the respective AF training set. We then remove the adapters with lowest activations.

Figure 5 demonstrates that we can remove most adapters in AF without affecting the task performance. With two remaining adapters, we achieve comparable results to the full AF models with eight adapters and improve the inference speed by $68 \%$.

We therefore recommend performing AdaperFusion pruning before deploying these models in practice. This is a simple yet effective technique

\footnotetext{
${ }^{10}$ We include detailed measurements in Appendix G.4.
} 
to achieve efficiency gains even when aiming at maintaining performance entirely.

\section{Conclusion}

Adapters have emerged as a suitable alternative to full model fine-tuning, and their most widely claimed computational advantage is the small model size. In this work, we have demonstrated that the advantages of adapters go far beyond mere parameter efficiency. Even without our extensions, the training steps of two common adapter architectures are up to $60 \%$ faster. However, these improvements come at the cost of 4-6\% slower inference speed. Thus, if training is more important, adapters can be advantageous over full model fine-tuning.

AdapterDrop expands these advantages by dropping a variable number of adapters from lower transformer layers. We dynamically reduce the computational overhead at run-time when performing inference over multiple tasks and maintain task performances to a large extent. This benefits researchers working on models that need to make multiple independent predictions on a single input.

Finally, we also investigated the computational efficiency of AdapterFusion models. We find that dropping entire AdapterFusion layers comes at a considerable performance/efficiency tradeoff, whereas pruning of the least activated adapters in each layer can improve the model efficiency while maintaining performance entirely.

We believe that our work can be widely extended and that there exist many more directions to obtain efficient adapter-based models. For instance, we could explore more efficient pre-trained adapters, ${ }^{11}$ sharing the adapter weights across layers, ${ }^{12}$ or pruning adapters from AdapterFusion at training time. ${ }^{13}$ In the Appendix to this paper, we present preliminary results for several related ideas, which may serve as a starting point for future work.

\section{Acknowledgments}

This work has received financial support from multiple sources. (1) The German Federal Ministry of

\footnotetext{
${ }^{11}$ In Appendix B, we evaluate MLM pre-trained adapters. Our results suggest that different strategies are necessary for adapters as compared to fully fine-tuned transformers, which can serve as a starting point for further experiments.

${ }^{12}$ Appendix D shows that adapter with shared weights across layers achieves comparable results to a standard adapter while drastically reducing the number of parameters.

${ }^{13}$ Appendix E shows that we can randomly dropout $75 \%$ of the adapters during AdapterFusion training with a minimal impact on the task performance.
}

Education and Research and the Hessian Ministry of Higher Education, Research, Science and the Arts within their joint support of the National Research Center for Applied Cybersecurity ATHENE. (2) The European Regional Development Fund (ERDF) and the Hessian State Chancellery - Hessian Minister of Digital Strategy and Development under the promotional reference 20005482 (TexPrax). (3) The German Research Foundation (DFG) as part of the Research Training Group KRITIS No. GRK 2222. (4) The German Federal Ministry of Education and Research (BMBF) as part of the Software Campus program under the promotional reference 01IS17050. (5) The LOEWE initiative (Hesse, Germany) within the emergenCITY center. (6) The German Research Foundation (DFG) as part of the UKP-SQuARE project (grant GU 798/29-1). Finally, we gratefully acknowledge the support of NVIDIA Corporation with the donation of the Titan X Pascal GPU used for this research.

\section{References}

Alan Ansell, Edoardo Maria Ponti, Jonas Pfeiffer, Sebastian Ruder, Goran Glavaš, Ivan Vulić, and Anna Korhonen. 2021. MAD-G: Multilingual Adapter Generation for Efficient Cross-Lingual Transfer. In Findings of the Association for Computational Linguistics: EMNLP 2021.

Haoli Bai, Wei Zhang, Lu Hou, Lifeng Shang, Jin Jin, Xin Jiang, Qun Liu, Michael Lyu, and Irwin King. 2021. BinaryBERT: Pushing the limit of BERT quantization. In Proceedings of the 59th Annual Meeting of the Association for Computational Linguistics and the 11th International Joint Conference on Natural Language Processing (ACL 2021), pages 4334-4348.

Ankur Bapna and Orhan Firat. 2019. Simple, Scalable Adaptation for Neural Machine Translation. In Proceedings of the 2019 Conference on Empirical Methods in Natural Language Processing and the 9th International Joint Conference on Natural Language Processing (EMNLP 2019), pages 1538-1548.

Tom B. Brown, Benjamin Mann, Nick Ryder, Melanie Subbiah, Jared Kaplan, Prafulla Dhariwal, Arvind Neelakantan, Pranav Shyam, Girish Sastry, Amanda Askell, Sandhini Agarwal, Ariel Herbert-Voss, Gretchen Krueger, Tom Henighan, Rewon Child, Aditya Ramesh, Daniel M. Ziegler, Jeffrey Wu, Clemens Winter, Christopher Hesse, Mark Chen, Eric Sigler, Mateusz Litwin, Scott Gray, Benjamin Chess, Jack Clark, Christopher Berner, Sam McCandlish, Alec Radford, Ilya Sutskever, and Dario Amodei. 2020. Language Models are Few-Shot Learners. arXiv preprint arXiv:2005.14165. 
Jacob Devlin, Ming-Wei Chang, Kenton Lee, and Kristina Toutanova. 2019. BERT: Pre-training of Deep Bidirectional Transformers for Language Understanding. In Proceedings of the 2019 Conference of the North American Chapter of the Association for Computational Linguistics: Human Language Technologies (NAACL 2019), pages 4171-4186.

Maha Elbayad, Jiatao Gu, Edouard Grave, and Michael Auli. 2020. Depth-adaptive transformer. In 8th International Conference on Learning Representations (ICLR 2020).

Angela Fan, Edouard Grave, and Armand Joulin. 2020. Reducing Transformer Depth on Demand with Structured Dropout. In 8th International Conference on Learning Representations, (ICLR 2020).

Lu Hou, Zhiqi Huang, Lifeng Shang, Xin Jiang, Xiao Chen, and Qun Liu. 2020. Dynabert: Dynamic BERT with adaptive width and depth. In 34th Conference on Neural Information Processing Systems (NeurIPS 2020).

Neil Houlsby, Andrei Giurgiu, Stanislaw Jastrzkebski, Bruna Morrone, Quentin de Laroussilhe, Andrea Gesmundo, Mona Attariyan, and Sylvain Gelly. 2019. Parameter-Efficient Transfer Learning for NLP. In Proceedings of the 36th International Conference on Machine Learning (ICML 2019).

Jeremy Howard and Sebastian Ruder. 2018. Universal Language Model Fine-tuning for Text Classification. In Proceedings of the 56th Annual Meeting of the Association for Computational Linguistics, (ACL 2018), pages 328-339.

Zhenzhong Lan, Mingda Chen, Sebastian Goodman, Kevin Gimpel, Piyush Sharma, and Radu Soricut. 2020. ALBERT: A Lite BERT for Self-supervised Learning of Language Representations. In 8th International Conference on Learning Representations (ICLR 2020).

Anne Lauscher, Olga Majewska, Leonardo F. R Ribeiro, Iryna Gurevych, Nikolai Rozanov, and Goran Glavaš. 2020. Common sense or world knowledge? investigating adapter-based knowledge injection into pretrained transformers. In Proceedings of Deep Learning Inside Out (DeeLIO): The First Workshop on Knowledge Extraction and Integration for Deep Learning Architectures, pages $43-$ 49.

Yinhan Liu, Myle Ott, Naman Goyal, Jingfei Du, Mandar Joshi, Danqi Chen, Omer Levy, Mike Lewis, Luke Zettlemoyer, and Veselin Stoyanov. 2019. RoBERTa: A Robustly Optimized BERT Pretraining Approach . arXiv preprint arXiv:1907.11692.

Sinno Jialin Pan and Qiang Yang. 2010. A Survey on Transfer Learning. IEEE Transactions on Knowledge and Data Engineering, 22(10):1345-1359.
Matthew E. Peters, Mark Neumann, Mohit Iyyer, Matt Gardner, Christopher Clark, Kenton Lee, and Luke Zettlemoyer. 2018. Deep Contextualized Word Representations. In Proceedings of the 2018 Conference of the North American Chapter of the Association for Computational Linguistics: Human Language Technologies, (NAACL 2018), pages 2227-2237.

Jonas Pfeiffer, Aishwarya Kamath, Andreas Rücklé, Kyunghyun Cho, and Iryna Gurevych. 2021a. AdapterFusion: Non-Destructive Task Composition for Transfer Learning. In Proceedings of the 16th Conference of the European Chapter of the Association for Computational Linguistics (EACL 2021), pages 487-503.

Jonas Pfeiffer, Andreas Rücklé, Clifton Poth, Aishwarya Kamath, Ivan Vulić, Sebastian Ruder, Kyunghyun Cho, and Iryna Gurevych. 2020a. AdapterHub: A Framework for Adapting Transformers. In Proceedings of the 2020 Conference on Empirical Methods in Natural Language Processing (EMNLP 2020): Systems Demonstrations, pages 4654.

Jonas Pfeiffer, Ivan Vulić, Iryna Gurevych, and Sebastian Ruder. 2020b. MAD-X: An Adapter-based Framework for Multi-task Cross-lingual Transfer. In Proceedings of the 2020 Conference on Empirical Methods in Natural Language Processing (EMNLP 2020), pages 7654-7673.

Jonas Pfeiffer, Ivan Vulić, Iryna Gurevych, and Sebastian Ruder. 2021b. UNKs Everywhere: Adapting Multilingual Language Models to New Scripts. In Proceedings of the 2021 Conference on Empirical Methods in Natural Language Processing, EMNLP 2021, Online, November, 2021.

Clifton Poth, Jonas Pfeiffer, Andreas Rücklé, and Iryna Gurevych. 2021. What to pre-train on? efficient intermediate task selection. In Proceedings of the 2021 Conference on Empirical Methods in Natural Language Processing (EMNLP 2021).

Alec Radford, Karthik Narasimhan, Tim Salimans, and Ilya Sutskever. 2018. Improving Language Understanding by Generative Pre-Training. Technical report, OpenAI.

Alec Radford, Jeffrey Wu, Rewon Child, David Luan, Dario Amodei, and Ilya Sutskever. 2019. Language Models are Unsupervised Multitask Learners. Technical report, OpenAI.

Andreas Rücklé, Jonas Pfeiffer, and Iryna Gurevych. 2020. MultiCQA: Exploring the Zero-Shot Transfer of Text Matching Models on a Massive Scale. In Proceedings of the 2020 Conference on Empirical Methods in Natural Language Processing (EMNLP 2020), pages 2471-2486.

Sebastian Ruder. 2019. Neural Transfer Learning for Natural Language Processing. Ph.D. thesis, National University of Ireland, Galway. 
Victor Sanh, Lysandre Debut, Julien Chaumond, and Thomas Wolf. 2019. DistilBERT, a distilled version of BERT: smaller, faster, cheaper and lighter. arXiv preprint arXiv:1910.01108.

Asa Cooper Stickland and Iain Murray. 2019. BERT and PALs: Projected Attention Layers for Efficient Adaptation in Multi-Task Learning. In Proceedings of the 36th International Conference on Machine Learning, (ICML 2019), pages 5986-5995.

Emma Strubell, Ananya Ganesh, and Andrew McCallum. 2019. Energy and Policy Considerations for Deep Learning in NLP. In Proceedings of the 57th Conference of the Association for Computational Linguistics, (ACL 2019), pages 3645-3650.

Zhiqing Sun, Hongkun Yu, Xiaodan Song, Renjie Liu, Yiming Yang, and Denny Zhou. 2020. MobileBERT: a Compact Task-Agnostic BERT for Resource-Limited Devices. In Proceedings of the 58th Annual Meeting of the Association for Computational Linguistics (ACL 2020), pages 2158-2170.

Lisa Torrey and Jude Shavlik. 2010. Transfer learning. In Handbook of research on machine learning applications and trends: algorithms, methods, and techniques, pages 242-264. IGI Global.

Ahmet Üstün, Arianna Bisazza, Gosse Bouma, and Gertjan van Noord. 2020. UDapter: Language Adaptation for Truly Universal Dependency Parsing. In Proceedings of the 2020 Conference on Empirical Methods in Natural Language Processing (EMNLP 2020), pages 2302-2315.

Marko Vidoni, Ivan Vulić, and Goran Glavaš. 2020. Orthogonal language and task adapters in zero-shot cross-lingual transfer. arXiv preprint arXiv:2012.06460.

Ivan Vulić, Edoardo Maria Ponti, Robert Litschko, Goran Glavaš, and Anna Korhonen. 2020. Probing Pretrained Language Models for Lexical Semantics. In Proceedings of the 2020 Conference on Empirical Methods in Natural Language Processing (EMNLP 2020), pages 7222-7240.

Alex Wang, Amanpreet Singh, Julian Michael, Felix Hill, Omer Levy, and Samuel R. Bowman. 2018. GLUE: A Multi-Task Benchmark and Analysis Platform for Natural Language Understanding. In Proceedings of the 2018 EMNLP Workshop BlackboxNLP: Analyzing and Interpreting Neural Networks for NLP, pages 353-355.

Ruize Wang, Duyu Tang, Nan Duan, Zhongyu Wei, Xuanjing Huang, Jianshu Ji, Guihong Cao, Daxin Jiang, and Ming Zhou. 2021. K-Adapter: Infusing Knowledge into Pre-Trained Models with Adapters. In Findings of the Association for Computational Linguistics: ACL-IJCNLP 2021, pages 1405-1418.

Wenhui Wang, Furu Wei, Li Dong, Hangbo Bao, Nan Yang, and Ming Zhou. 2020. Minilm: Deep selfattention distillation for task-agnostic compression of pre-trained transformers. In 34th Conference on Neural Information Processing Systems (NeurIPS 2020).

Ji Xin, Raphael Tang, Jaejun Lee, Yaoliang Yu, and Jimmy Lin. 2020. Deebert: Dynamic early exiting for accelerating BERT inference. In Proceedings of the 58th Annual Meeting of the Association for Computational Linguistics (ACL 2020), pages 22462251.

\section{A Measuring Computational and Task Performance}

\section{A.1 Computational Efficiency}

We use Python 3.6, PyTorch 1.5.1, CUDA 10.1 for all measurements. We repeat them with two different GPUs: NVIDIA Tesla V100 PCIe (32GB) and a NVIDIA Titan X Pascal (12GB). We make use of the torch. cuda. Event class and torch.cuda.synchronize to measure only the exact period of time of a training (or inference) step. ${ }^{14}$ For both inference and training, we repeat the respective step 300 times. We report the median to mitigate the impact of outliers caused by GPU warmup.

Relativ speed. We define the relative speed of an adapter compared full model fine-tuning as: $\frac{S_{a}}{S_{f}}$ where $S_{a}$ and $S_{f}$ are the time of one step with the adapter model and the fully fine-tuned model, respectively. For example, a relative speed of 1.5 means that the adapter model can perform 1.5 steps in the time the fully fine-tuned model performs one step.

Speedup. Speedup describes the positive change in relative speed of an adapter model when using AdapterDrop (or another method). A speedup of $p \%$ means that the adapter model with AdapterDrop requires only $(1-p / 100) \times$ of the runtime than the adapter model without AdapterDrop.

The speedup of AdapterDrop (and AdapterFusion) are additive. If dropping one layer results in $p \%$ speedup, dropping two layers results in $2 p \%$ speedup, etc.

\section{A.2 Task Performances}

We study the task performances of adapter models on the popular GLUE benchmark (Wang et al., 2018). Following Devlin et al. (2019), we exclude

\footnotetext{
${ }^{14}$ This is necessary due to the asynchronous nature of the command execution on CPU and GPU.
} 
the WNLI because of the problematic data construction. ${ }^{15}$ We perform our analyses using RoBERTa base (Liu et al., 2019) as our pre-trained model and report the mean and standard deviation over three runs of the best development performance evaluated after every epoch. We train larger data sets (SST-2, MNLI, QNLI, and QQP) for 10 epochs and the rest of the data sets for 20 epochs. We use a batch size of 32 and, if not otherwise noted, the default hyperparameters for adapter fine-tuning as in (Pfeiffer et al., 2021a).

\section{B Adapter Initialization and Convergence}

Besides measuring training and inference time, we are interested in (1) how using adapters compare to standard RoBERTa-base with regards to downstream task convergence, and (2) if initializing adapters with pre-trained weights using masked language modeling can lead to faster convergence.

First, we compare RoBERTa-base with adapter models using the architecture proposed by Pfeiffer et al. (2021a). Second, we pretrain an adapter with masked language modeling (MLM) using documents from the English Wikipedia. ${ }^{16}$ The results for both experiments are visualized in Figure 12. When comparing RoBERTa-base with randomly initialized adapters, We find that adapters do not come at the cost of requiring more training steps for convergence (1). For several of the eight GLUE tasks, we observe similar convergence behavior with the standard RoBERTa-base model and its counterpart using adapters.

Further, we observe across all tasks that initializing the adapter weights with MLM pre-training does not have a substantial impact on the downstream task convergence (compared to a randomly initialized adapter). Thus, we find no evidence that pre-training of adapters with our masked language modeling objective leads to better convergence performance in our experiments (2).

\section{Detailed Results: AdapterDrop Task Performances}

We plot the detailed task performances of AdapterDrop with the different training strategies in Figure 13. The relative differences of AdapterDrop to

\footnotetext{
${ }^{15}$ See https : / / gluebenchmark . com/faq

${ }^{16}$ We used a recent dump of English Wikipedia. We train with a batch size of 64 and for 250k steps such that no sentence was used twice.
}

a standard adapter with no AdapterDrop are given in Table 8.

\section{Adapter with Cross-Layer Parameter Sharing}

We can further reduce the number of parameters required for each task by sharing the weights of the adapters across all transformer layers. This is similar to weight sharing in ALBERT (Lan et al., 2020), but specialized on adapters and can therefore be applied to a wide range of pre-trained models.

We use the Pfeiffer adapter architecture in our experiments with the same hyperparameters as in Appendix A.2. Because cross-layer parameter sharing reduces the capacity of adapter models, we study the impact of the adapter compression rate. The compression rate refers to the down-projection factor in the adapter's bottleneck layer and thus impacts the its capacity (the compression rate specifies by how much 'FF Down' in Figure 1 compresses the representations). The standard compression rate is 16 , and smaller values result in a larger model capacity.

Table 6 shows that cross-layer parameter sharing with the same compression rate of 16 largely maintains the performance compared to separate weights with an average difference of $2.35 \%$. With a smaller compression rate of 4 , we close this gap by more than $50 \%$ while still requiring $66 \%$ fewer parameters. ${ }^{17}$ The resulting models are lightweight: our shared adapter with a compression rate of 16 requires only $307 \mathrm{~KB}$ storage space.

\section{E Training AdapterFusion with Dropout}

We investigate the random dropout of adapters from AdapterFusion during training (using our eight task adapters as in §4) to improve the speed of training steps. Each layer randomly selects different adapters to drop out. This means that the model itself may still use the knowledge from all tasks, although not in the layers individually.

Table 7 shows the results for the four smallest GLUE tasks in terms of training data size. The speedup that we achieve with AdapterFusion dropout can be substantial: with a dropout rate of $75 \%$ (i.e., dropping out 6 out of our 8 adapters) each training step is $74 \%$ faster on average (with a sequence length of 128 , a batch size of 32). We observe no clear trend in terms of task performances. Fusion dropout leads to consistent decreases on

\footnotetext{
${ }^{17}$ Even smaller compression rates do not yield similar gains.
} 
RTE and CoLA, only a small impact on STS-B (no difference when dropping out $25 \%$ of adapters), and yields improvements on MRPC.

The effectiveness of Fusion dropout, thus, depends on the individual downstream task. Nevertheless, we believe that this methods could be suitable, e.g., for resource-constrained settings.

\section{F Detailed Results: Removing AdapterFusion Layers}

The computational overhead of AF can be reduced during inference by decreasing the number of adapters. We investigate how dropping AF layers impacts the performance on the four smallest GLUE tasks (MRPC, STS-B, CoLA, RTE) and visualize the results in Figure 7.

In this experiment we compare the performance of AF with and without AdapterDrop during training. For both, we use standard adapters as well as adapters created via AdapterDrop as basis for AF. Unsurprisingly, the performance of AF without AdapterDrop within the adapters or fusion drops fastest on all four datasets. Using AdapterDrop when creating the adapters, applying AdapterDrop on AF, or the combination of both significantly reduces the performance drop when omitting fusion layers during inference. On RTE and MRPC, multiple AF layers can be omitted while still performing en par with or better compared to a single task adapter. We further find this robustness to be task dependent. Even AF with AdapterDrop shows a steep fall in performance on RTE and CoLA, while being relatively stable on MRPC and STS-B, even with most layers omitted.

\section{G Detailed Efficiency Measurements}

In this section, we present detailed results of our efficiency measurements for V100 and TitanX GPUs.

\section{G.1 Adapters}

We present the efficiency results for adapters and fully fine-tuned models in Figure 6, where we plot the required time (absolute numbers) during training and inference. The relative speed of adapters compared to fully fine-tuned models is given in Table 9.

\section{G.2 AdapterDrop}

Multi-task inference. In Figure 8, we plot the speed of adapters in a multi-task setting compared to fully fine-tuned models with sequential processing of inputs. In Table 11, we present the relative speed of adapters in this setting and show the speedup gained with AdapterDrop for each dropped layer. The average speedup in Table 2 is calculated as the average speedup over the batch sizes 16, 32 and 64 in Table 11.

Training adapters with dropped layers. Table 5 shows the speedup of AdapterDrop when training a single adapter. The average speedup for training with AdapterDrop is $4.7 \%$ per layer for the V100 and $4.5 \%$ for the TitanX. This is the average result over batch sizes 16, 32, 64 and sequence length 64 , 128, 256, and 256 (see Table 5).

\section{G.3 AdapterFusion}

We plot the speed of AdapterFusion with different numbers of included adapters in Figure 9. In Table 10, we present the relative speed of AdapterFusion compared to a fully-finetuned model and a model with one adapter. This also shows the computational overhead (slowdown) that results from adding more adapters to AdapterFusion.

\section{G.4 AdapterDrop for AdapterFusion}

Table 4 shows the speedup gained with AdapterDrop for AdapterFusion during training and inference. Figure 10 shows the required time as a function of the dropped layers.

\section{H Parallel Implementation of AdapterFusion}

AdapterHub's implementation of AdapterFusion passes through each task adapter sequentially. We hypothesized that a better efficiency can be achieved with parallel processing of adapters. We implement the parallel computation of the different adapters by reformulation the linear layers as two convolutions.

The first convolution is a convolution with a kernel size equal to the hidden dimension of the transformer and output channels equal to the number of adapters times the downprojection dimension of the adapters. The second convolution is a grouped convolution ${ }^{18}$ which processes the channels in blocks the size of the downprojection dimension. It outputs channels equal to the number of adapters times the hidden dimension.

\footnotetext{
${ }^{18}$ Using the 'groups' parameter in Pytorch (https: //pytorch.org/docs/stable/generated/ torch.nn. Convld.html\#torch.nn. Convld)
} 


\begin{tabular}{|c|c|c|c|c|}
\hline \multirow[b]{3}{*}{ Adapters } & \multicolumn{4}{|c|}{ Speedup (per dropped layer) } \\
\hline & \multicolumn{2}{|c|}{ Inference } & \multicolumn{2}{|c|}{ Training } \\
\hline & V100 & TitanX & V100 & $\operatorname{Titan} X$ \\
\hline 2 & $3.0 \%$ & $3.1 \%$ & $6.3 \%$ & $6.4 \%$ \\
\hline 4 & $4.0 \%$ & $4.1 \%$ & $6.8 \%$ & $6.8 \%$ \\
\hline 8 & $5.2 \%$ & $5.2 \%$ & $7.3 \%$ & $7.3 \%$ \\
\hline 16 & $6.3 \%$ & $6.3 \%$ & $7.8 \%$ & - \\
\hline
\end{tabular}

Table 4: The speedup for each dropped layer for AdapterFusion during training and inference. Measurements were conducted with a batch size of 32 and sequence length of 128 . Missing values are due to insufficient GPU memory.

\begin{tabular}{rrrr}
\hline & & \multicolumn{2}{c}{ Speedup } \\
\cline { 3 - 4 } Batch Size & Seq. Len & V100 & TitanX \\
\hline 16 & 64 & $4.6 \%$ & $4.4 \%$ \\
16 & 128 & $4.6 \%$ & $4.6 \%$ \\
16 & 256 & $4.8 \%$ & $4.6 \%$ \\
16 & 512 & $4.7 \%$ & - \\
32 & 64 & $4.6 \%$ & $4.5 \%$ \\
32 & 128 & $4.7 \%$ & $4.5 \%$ \\
32 & 256 & $4.6 \%$ & $4.7 \%$ \\
32 & 512 & $4.8 \%$ & - \\
64 & 64 & $4.7 \%$ & $4.5 \%$ \\
64 & 128 & $4.6 \%$ & $4.5 \%$ \\
64 & 256 & $4.7 \%$ & - \\
64 & 512 & - & - \\
\hline
\end{tabular}

Table 5: Speedup for each dropped layer during training with AdapterDrop on the V100 and TitanX.

We show in Figure 11 and in Table 12 that the iterative implementation is faster than the parallel implementation for larger input sizes (e.g., batch sizes greater than). This indicates that once the input can no longer be processed entirely in parallel on the GPU (due to limited CUDA cores) the iterative implementation seems to be more efficient.

\begin{tabular}{lrrrrr}
\hline & Standard & \multicolumn{3}{c}{ Cross-Layer Parameter Sharing } \\
\cline { 5 - 6 } \cline { 5 - 6 } Compression rate $=16$ & & 1.33 & 4 & 16 \\
\hline SST-2 & $94.7 \pm 0.3$ & $94.2 \pm 0.3$ & $94.2 \pm 0.1$ & $94.1 \pm 0.4$ \\
QNLI & $93.0 \pm 0.2$ & $92.4 \pm 0.1$ & $93.1 \pm 0.1$ & $90.6 \pm 1.4$ \\
MNLI & $87.3 \pm 0.1$ & $87.0 \pm 0.1$ & $87.1 \pm 0.0$ & $86.2 \pm 0.2$ \\
QQP & $90.6 \pm 0.0$ & $90.8 \pm 0.1$ & $90.2 \pm 0.0$ & $88.6 \pm 0.5$ \\
CoLA & $62.6 \pm 0.9$ & $60.3 \pm 1.6$ & $60.8 \pm 0.4$ & $57.2 \pm 1.0$ \\
MRPC & $88.4 \pm 0.1$ & $88.2 \pm 0.7$ & $88.5 \pm 1.1$ & $86.8 \pm 0.5$ \\
RTE & $75.9 \pm 2.2$ & $69.4 \pm 0.5$ & $71.5 \pm 2.7$ & $71.5 \pm 1.0$ \\
STS-B & $90.3 \pm 0.1$ & $89.5 \pm 0.1$ & $89.7 \pm 0.3$ & $89.0 \pm 0.7$ \\
\hline Average & 85.35 & 83.98 & 84.39 & 83.0 \\
\hline Params & $884 k$ & $884 k$ & $295 k$ & $74 k$ \\
\hline
\end{tabular}

Table 6: Task performance scores of the standard approach with separate adapter weights vs. cross-layer parameter sharing. The compression rate denotes the factor by which 'FF Down' in Figure 1 compresses the representations. The number of parameters is given without classification heads.

\begin{tabular}{lrrrr}
\hline & \multicolumn{4}{c}{ Fusion Dropout } \\
\cline { 2 - 5 } & \multicolumn{1}{c}{$0 \%$} & \multicolumn{1}{c}{$25 \%$} & \multicolumn{1}{c}{$50 \%$} & \multicolumn{1}{c}{$75 \%$} \\
\hline CoLA & $63.9 \pm 0.6$ & $62.9 \pm 0.8$ & $62.4 \pm 0.7$ & $60.4 \pm 0.2$ \\
MRPC & $88.4 \pm 0.1$ & $89.2 \pm 0.5$ & $89.2 \pm 0.4$ & $89.3 \pm 0.1$ \\
RTE & $85.4 \pm 0.7$ & $82.8 \pm 1.9$ & $82.1 \pm 0.3$ & $80.9 \pm 1.1$ \\
STS-B & $90.2 \pm 0.1$ & $90.2 \pm 0.1$ & $90.1 \pm 0.1$ & $89.9 \pm 0.1$ \\
\hline Speedup (8) & - & $15.9 \%$ & $39.4 \%$ & $73.7 \%$ \\
Speedup (16) & - & $22.5 \%$ & $58.2 \%$ & $120.6 \%$ \\
\hline
\end{tabular}

Table 7: Development scores of AdapterFusion (compression rate 16x) with or without fusion dropout during training. Fusion dropout of $50 \%$ means that each adapter has a $50 \%$ chance of not being used as input to the fusion layer. The speedup depends on the total number of adapters used in AdapterFusion (8 adapters in our setting here, 16 used by Pfeiffer et al. (2021a)) 


\begin{tabular}{lrrrrrrrrrrrr}
\hline & \multicolumn{11}{c}{ Dropped Layers } \\
\cline { 2 - 12 } & 0 & 1 & 2 & 3 & 4 & 5 & 6 & 7 & 8 & 9 & 10 & 11 \\
\hline Standard adapter & 100.0 & 98.5 & 97.1 & 95.3 & 92.0 & 89.0 & 82.2 & 74.6 & 64.5 & 54.5 & 49.3 & 43.3 \\
\hline Specialized AdapterDrop (12 models) & 100.0 & 99.5 & 98.9 & 98.2 & 97.6 & 97.1 & 95.9 & 95.3 & 95.1 & 94.3 & 92.5 & 82.9 \\
Robust AdapterDrop & 98.5 & 97.7 & 97.3 & 96.8 & 96.1 & 95.4 & 94.5 & 93.3 & 92.2 & 89.9 & 85.9 & 62.0 \\
\hline
\end{tabular}

Table 8: Model performances with AdapterDrop in relation to a standard adapter with no dropped layers. We report the percentage of retained task performance compared to the standard adapter with no dropped layers during evaluation. The results are averaged over all eight GLUE task. A value of 97.1 for specialized AdapterDrop with five dropped layers means that the model achieves $97.1 \%$ of the performance compared to the standard adapter with no dropped layers. Performance scores for each task can be found in Figure 13.

\begin{tabular}{|c|c|c|c|c|c|c|c|c|c|}
\hline \multirow{3}{*}{ Sequence Len. } & \multirow{3}{*}{ Batch Size } & \multicolumn{4}{|c|}{ V100 } & \multicolumn{4}{|c|}{ TitanX } \\
\hline & & \multicolumn{2}{|c|}{ Training } & \multicolumn{2}{|c|}{ Inference } & \multicolumn{2}{|c|}{ Training } & \multicolumn{2}{|c|}{ Inference } \\
\hline & & Houlsby & Pfeiffer & Houlsby & Pfeiffer & Houlsby & Pfeiffer & Houlsby & Pfeiffer \\
\hline 64 & 16 & 0.98 & 1.70 & 0.92 & 0.94 & 1.61 & 1.69 & 0.93 & 0.94 \\
\hline 64 & 32 & 1.70 & 1.81 & 0.94 & 0.95 & 1.48 & 1.55 & 0.93 & 0.94 \\
\hline 64 & 64 & 1.46 & 1.54 & 0.94 & 0.95 & 1.40 & 1.46 & 0.94 & 0.94 \\
\hline 64 & 128 & 1.48 & 1.55 & 0.95 & 0.96 & 1.37 & 1.42 & 0.94 & 0.94 \\
\hline 128 & 16 & 1.48 & 1.57 & 0.94 & 0.95 & 1.45 & 1.52 & 0.93 & 0.94 \\
\hline 128 & 32 & 1.53 & 1.60 & 0.94 & 0.95 & 1.38 & 1.44 & 0.94 & 0.95 \\
\hline 128 & 64 & 1.47 & 1.53 & 0.95 & 0.96 & 1.35 & 1.40 & 0.94 & 0.95 \\
\hline 128 & 128 & 1.42 & 1.48 & 0.95 & 0.96 & - & - & - & - \\
\hline 256 & 16 & 1.42 & 1.49 & 0.94 & 0.95 & 1.34 & 1.38 & 0.94 & 0.95 \\
\hline 256 & 32 & 1.40 & 1.46 & 0.95 & 0.96 & 1.31 & 1.36 & 0.94 & 0.96 \\
\hline 256 & 64 & 1.40 & 1.45 & 0.95 & 0.96 & - & - & - & - \\
\hline 256 & 128 & - & - & - & - & - & - & - & - \\
\hline 512 & 16 & 1.36 & 1.41 & 0.96 & 0.96 & - & - & - & - \\
\hline 512 & 32 & 1.33 & 1.37 & 0.96 & 0.96 & - & - & - & - \\
\hline 512 & 64 & - & - & - & - & - & - & - & - \\
\hline 512 & 128 & - & - & - & - & - & - & - & - \\
\hline
\end{tabular}

Table 9: Relative speed of adapters compared to fully fine-tuned models. Missing values are due to insufficient GPU memory.

\begin{tabular}{|c|c|c|c|c|c|c|c|c|c|c|c|c|c|}
\hline \multirow[b]{3}{*}{ Seq. Len } & \multirow[b]{3}{*}{ Batch Size } & \multicolumn{6}{|c|}{ V100 } & \multicolumn{6}{|c|}{ TitanX } \\
\hline & & \multicolumn{2}{|c|}{ vs. FF } & \multicolumn{2}{|c|}{ vs. Adap. } & \multicolumn{2}{|c|}{ Slowdown } & \multicolumn{2}{|c|}{ vs. FF } & \multicolumn{2}{|c|}{ vs. Adap } & \multicolumn{2}{|c|}{ Slowdown } \\
\hline & & Tr. & Inf. & Tr. & Inf. & Tr. & Inf. & Tr. & Inf. & Tr. & Inf. & Tr. & Inf. \\
\hline 64 & 16 & 0.77 & 0.62 & 0.45 & 0.66 & $8.2 \%$ & $10.6 \%$ & 0.88 & 0.62 & 0.52 & 0.66 & $10.3 \%$ & $10.2 \%$ \\
\hline 64 & 32 & 1.03 & 0.64 & 0.57 & 0.68 & $12.0 \%$ & $11.1 \%$ & 0.80 & 0.61 & 0.52 & 0.64 & $11.2 \%$ & $11.0 \%$ \\
\hline 64 & 64 & 0.87 & 0.64 & 0.57 & 0.67 & $12.6 \%$ & $12.0 \%$ & 0.76 & 0.61 & 0.52 & 0.65 & $11.6 \%$ & $11.4 \%$ \\
\hline 128 & 16 & 0.91 & 0.65 & 0.58 & 0.69 & $12.0 \%$ & $11.0 \%$ & 0.80 & 0.61 & 0.53 & 0.65 & $10.9 \%$ & $10.8 \%$ \\
\hline 128 & 32 & 0.92 & 0.64 & 0.57 & 0.68 & $12.5 \%$ & $11.8 \%$ & 0.76 & 0.62 & 0.53 & 0.66 & $11.4 \%$ & $11.1 \%$ \\
\hline 128 & 64 & 0.87 & 0.65 & 0.57 & 0.68 & $12.5 \%$ & $11.6 \%$ & - & - & - & - & - & - \\
\hline 256 & 16 & 0.88 & 0.66 & 0.59 & 0.69 & $12.1 \%$ & $11.3 \%$ & 0.77 & 0.65 & 0.56 & 0.68 & $10.8 \%$ & $10.4 \%$ \\
\hline 256 & 32 & 0.86 & 0.68 & 0.59 & 0.70 & $11.9 \%$ & $11.3 \%$ & - & - & - & - & - & - \\
\hline 256 & 64 & - & - & - & - & - & - & - & - & - & - & - & - \\
\hline 512 & 16 & 0.87 & 0.69 & 0.62 & 0.72 & $11.2 \%$ & $10.1 \%$ & - & - & - & - & - & - \\
\hline 512 & 32 & - & - & - & - & - & - & - & - & - & - & - & - \\
\hline 512 & 64 & - & - & - & - & - & - & - & - & - & - & - & - \\
\hline
\end{tabular}

Table 10: Relative speed of AdapterFusion for different sequence lengths and batch sizes. We compute the training (Tr.) speed and inference (Inf.) speed with two adapters in AdapterFusion. We compare this to: FF, a fully fine-tuned model; Adap, an adapter model (Pfeiffer architecture). The slowdown denotes the computational overhead of each additional adapter composed in AdapterFusion (calculated as the average slowdown for adding one adapter to AF consisting of 2-16 adapters). Missing values are due to insufficient GPU memory. 

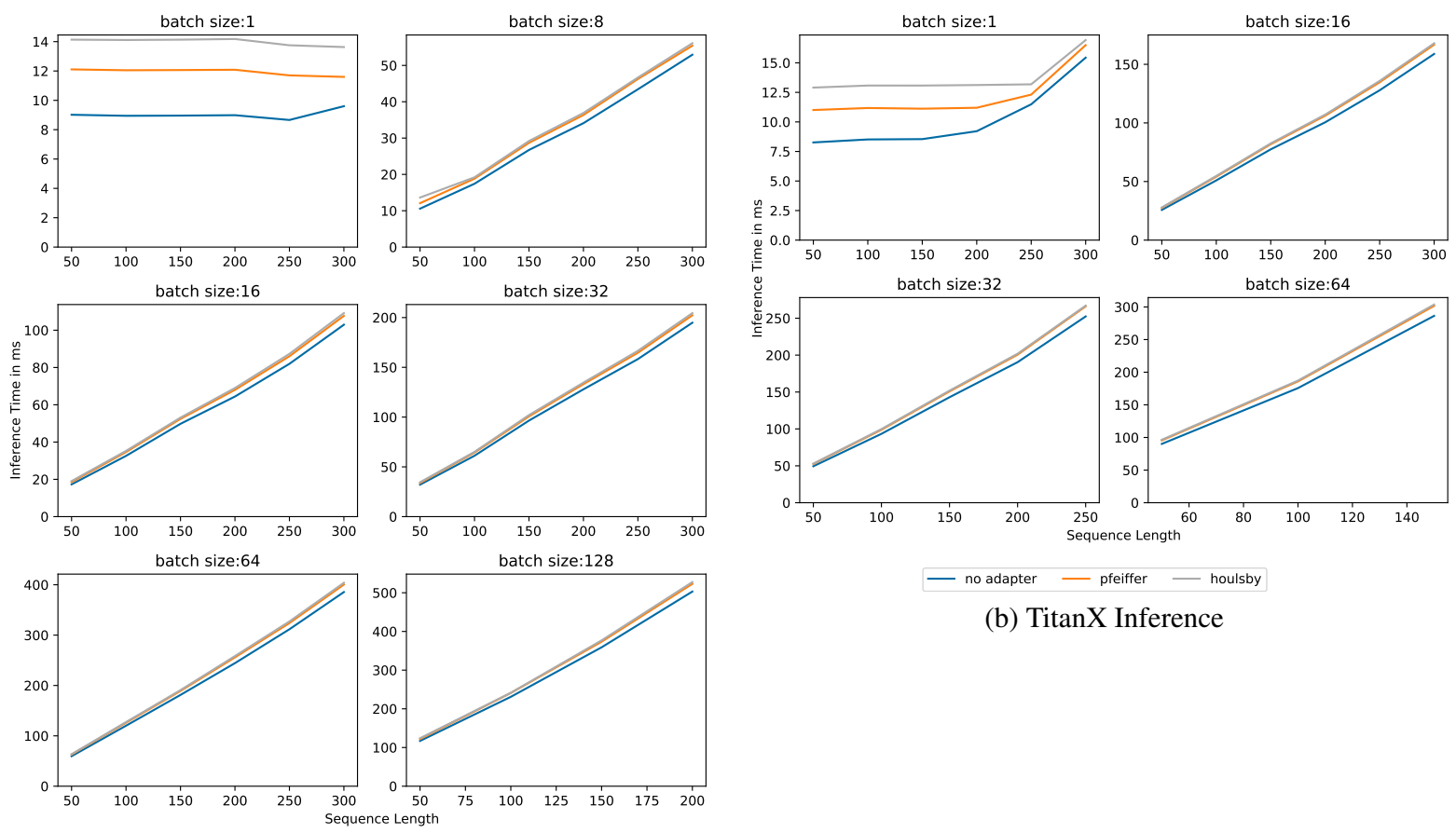

(b) TitanX Inference

— no adapter — pfeiffer - houlsby

(a) V100 Inference
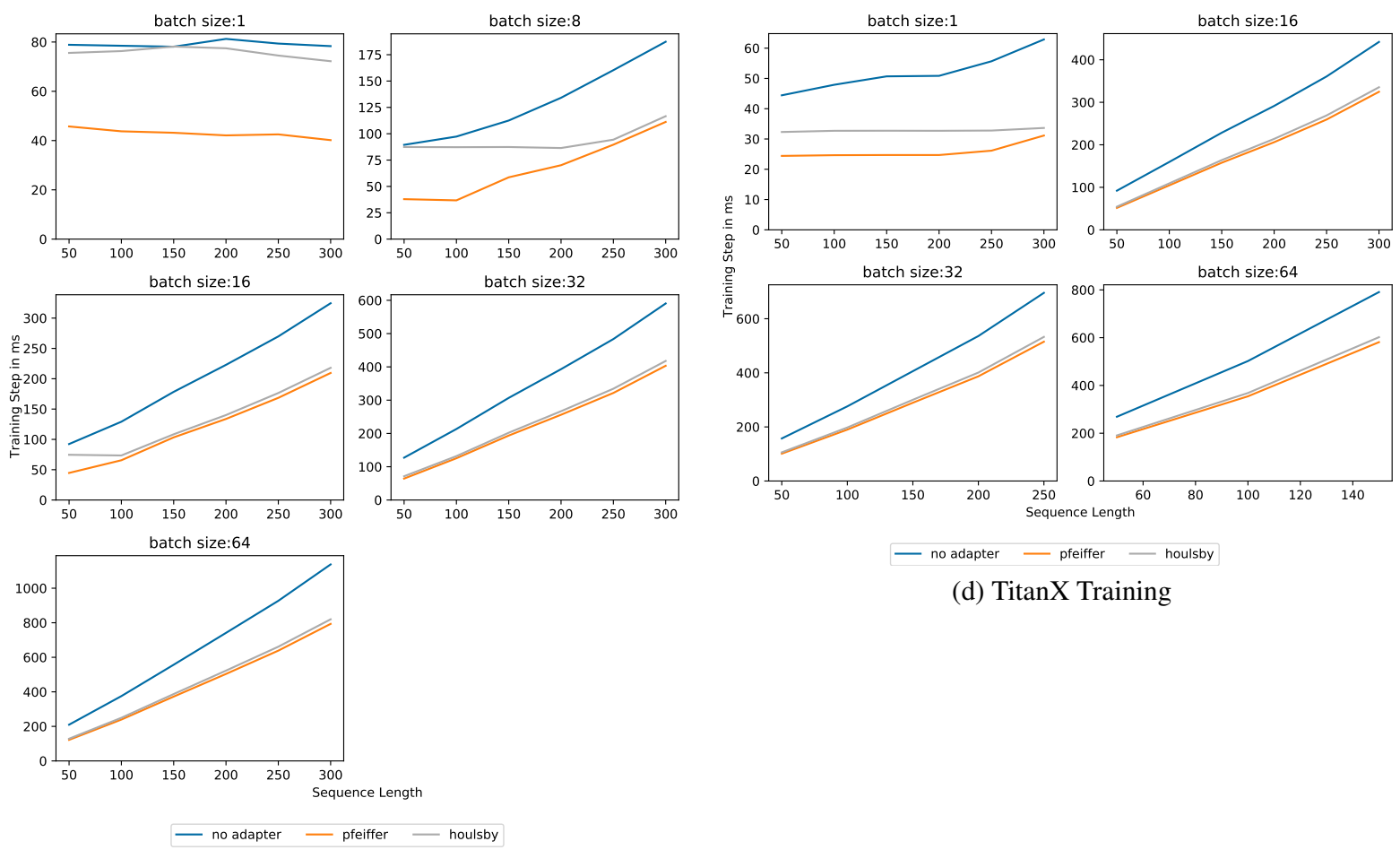

(d) TitanX Training

(c) V100 Training

Figure 6: The absolute time for each inference or training step. We compare a transformer model without adapters and an adapter model with Pfeiffer or Houlsby architectures. We note that for small inputs, i.e., batch size 1 or 8 , the time does not increase with the sequence length because the GPU is not working at capacity. Figure (b) with batch size 1 shows the transition from working under and working at capacity. 

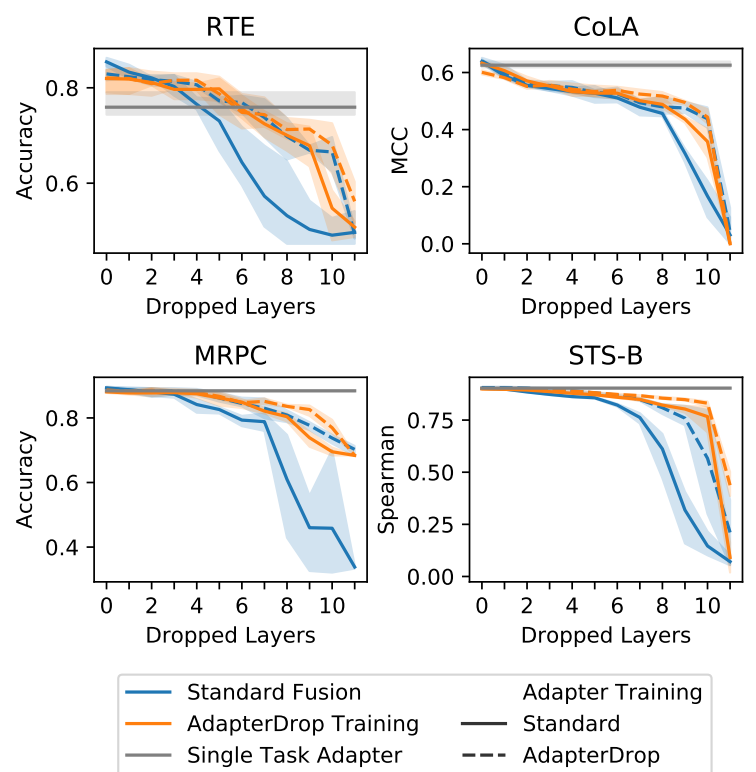
Adapter Training

ining - Standard - Single Task Adapter --- AdapterDrop

Figure 7: Performance of AF by the number of dropped AF layers. We show the results for AF and the used adapters (both with and without AdapterDrop), and compare the performance with a standard single task adapter.

\begin{tabular}{|c|c|c|c|c|}
\hline Device & Batch Size & Adapters & Inference & Speedup \\
\hline \multirow{16}{*}{ V100 } & 1 & 2 & 1.25 & $2.6 \%$ \\
\hline & 1 & 4 & 1.97 & $3.7 \%$ \\
\hline & 1 & 8 & 2.80 & $4.9 \%$ \\
\hline & 1 & 16 & 2.97 & $6.5 \%$ \\
\hline & 16 & 2 & 1.13 & $4.1 \%$ \\
\hline & 16 & 4 & 1.14 & $6.5 \%$ \\
\hline & 16 & 8 & 1.20 & $7.7 \%$ \\
\hline & 16 & 16 & 1.16 & $8.4 \%$ \\
\hline & 32 & 2 & 1.08 & $4.5 \%$ \\
\hline & 32 & 4 & 1.14 & $6.6 \%$ \\
\hline & 32 & 8 & 1.11 & $7.9 \%$ \\
\hline & 32 & 16 & 1.11 & $8.5 \%$ \\
\hline & 64 & 2 & 1.08 & $4.3 \%$ \\
\hline & 64 & 4 & 1.05 & $6.7 \%$ \\
\hline & 64 & 8 & 1.06 & $7.9 \%$ \\
\hline & 64 & 16 & 1.06 & $8.4 \%$ \\
\hline \multirow{4}{*}{ TitanX } & 32 & 2 & 1.07 & $4.4 \%$ \\
\hline & 32 & 4 & 1.09 & $6.6 \%$ \\
\hline & 32 & 8 & 1.09 & $7.8 \%$ \\
\hline & 32 & 16 & 1.06 & $8.4 \%$ \\
\hline \multirow{4}{*}{ CPU } & 1 & 2 & 0.98 & $4.2 \%$ \\
\hline & 1 & 4 & 1.03 & $6.5 \%$ \\
\hline & 1 & 8 & 1.05 & $7.7 \%$ \\
\hline & 1 & 16 & 1.06 & $8.4 \%$ \\
\hline
\end{tabular}

Table 11: The relative inference speed of simultaneous processing of multiple tasks with adapters compared to sequential processing of tasks with fully fine-tuned models. Gray columns show the speedup of AdapterDrop for every additional dropped layer. All measurements use a sequence length of 128 . Batch size 1 for the V100 is an outlier in both speedup and relative speed compared to the other results due to the small input size (compare with Figure 8).

\begin{tabular}{|c|c|c|c|c|}
\hline \multirow[b]{2}{*}{ Adapters } & \multirow[b]{2}{*}{ Seq. Len } & \multirow[b]{2}{*}{ Batch Size } & \multicolumn{2}{|c|}{ Rel. Speed } \\
\hline & & & V100 & TitanX \\
\hline 2 & 100 & 1 & 0.93 & 0.94 \\
\hline 3 & 100 & 1 & 0.89 & 0.88 \\
\hline 5 & 100 & 1 & 0.77 & 0.76 \\
\hline 10 & 100 & 1 & 0.60 & 1.29 \\
\hline 2 & 100 & 16 & 1.02 & 1.44 \\
\hline 3 & 100 & 16 & 1.12 & 1.58 \\
\hline 5 & 100 & 16 & 1.17 & 1.80 \\
\hline 10 & 100 & 16 & 1.27 & 2.14 \\
\hline 2 & 100 & 32 & 1.01 & 1.48 \\
\hline 3 & 100 & 32 & 1.17 & 1.62 \\
\hline 5 & 100 & 32 & 1.23 & 1.85 \\
\hline 10 & 100 & 32 & 1.32 & 2.24 \\
\hline 2 & 200 & 1 & 0.93 & 1.24 \\
\hline 3 & 200 & 1 & 0.88 & 1.37 \\
\hline 5 & 200 & 1 & 0.77 & 1.55 \\
\hline 10 & 200 & 1 & 0.52 & 1.87 \\
\hline 2 & 200 & 16 & 1.01 & 1.46 \\
\hline 3 & 200 & 16 & 1.17 & 1.59 \\
\hline 5 & 200 & 16 & 1.23 & 1.82 \\
\hline 10 & 200 & 16 & 1.32 & 2.21 \\
\hline 2 & 200 & 32 & 1.00 & 1.11 \\
\hline 3 & 200 & 32 & 1.18 & 1.17 \\
\hline 5 & 200 & 32 & 1.26 & - \\
\hline 10 & 200 & 32 & 1.34 & - \\
\hline 2 & 300 & 1 & 0.93 & 1.37 \\
\hline 3 & 300 & 1 & 0.88 & 1.50 \\
\hline 5 & 300 & 1 & 0.91 & 1.70 \\
\hline 10 & 300 & 1 & 0.94 & 2.03 \\
\hline 2 & 300 & 16 & 1.00 & 1.48 \\
\hline 3 & 300 & 16 & 1.16 & 1.63 \\
\hline 5 & 300 & 16 & 1.22 & 1.88 \\
\hline 10 & 300 & 16 & 1.32 & - \\
\hline 2 & 300 & 32 & 1.00 & - \\
\hline 3 & 300 & 32 & 1.20 & - \\
\hline 5 & 300 & 32 & 1.27 & - \\
\hline 10 & 300 & 32 & 1.36 & - \\
\hline 2 & 400 & 1 & 1.04 & 1.39 \\
\hline 3 & 400 & 1 & 1.09 & 1.51 \\
\hline 5 & 400 & 1 & 1.10 & 1.74 \\
\hline 10 & 400 & 1 & 1.10 & 2.08 \\
\hline 2 & 400 & 16 & 1.00 & - \\
\hline 3 & 400 & 16 & 1.18 & - \\
\hline 5 & 400 & 16 & 1.25 & - \\
\hline 10 & 400 & 16 & 1.34 & - \\
\hline 2 & 400 & 32 & 1.00 & - \\
\hline 3 & 400 & 32 & 1.20 & - \\
\hline 5 & 400 & 32 & 1.27 & - \\
\hline 10 & 400 & 32 & - & - \\
\hline
\end{tabular}

Table 12: Relative speed of AdapterFusion with the iterative implementation versus the parallel implementation with different batch sizes, sequence lengths and numbers of adapters for the V100 and TitanX. The parallel implementation is faster if the input is sufficiently small (batch size 1 or 2 adapters) as the GPU is not working at capacity and is able to use the parallel implementation. 

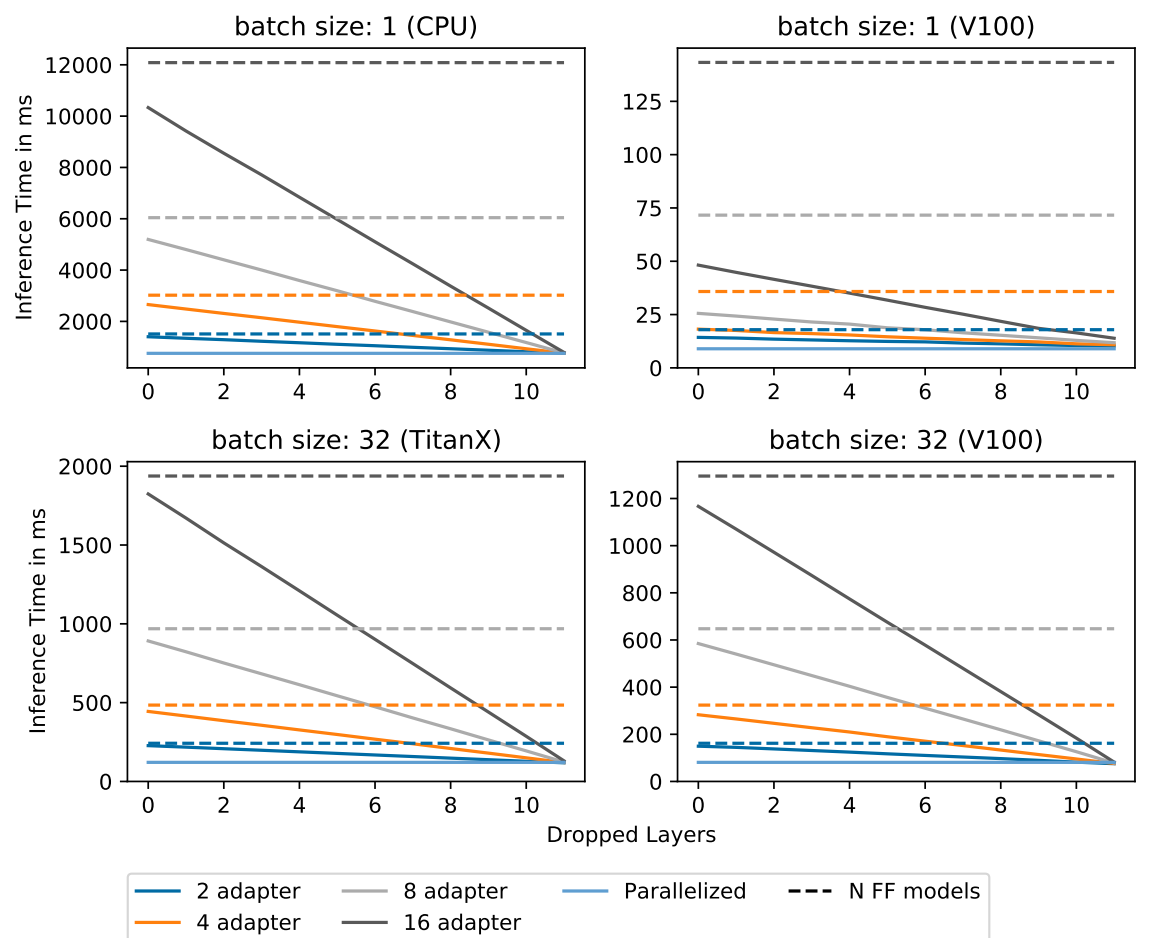

Figure 8: The absolute time required for performing inference for multiple tasks on the same input. The measurements are conducted with a sequence length of 128. N FF models denotes $N$ fully fine-tuned models, executed sequentially. Parallelized denotes the time required by $\mathrm{N}$ fully fine-tuned models running fully parallelized. Batch size 1 on the V100 is an outlier compared to the other results with a smaller speedup for each dropped layer but a higher relative speed compared to the fine-tuned models due to the small input size.

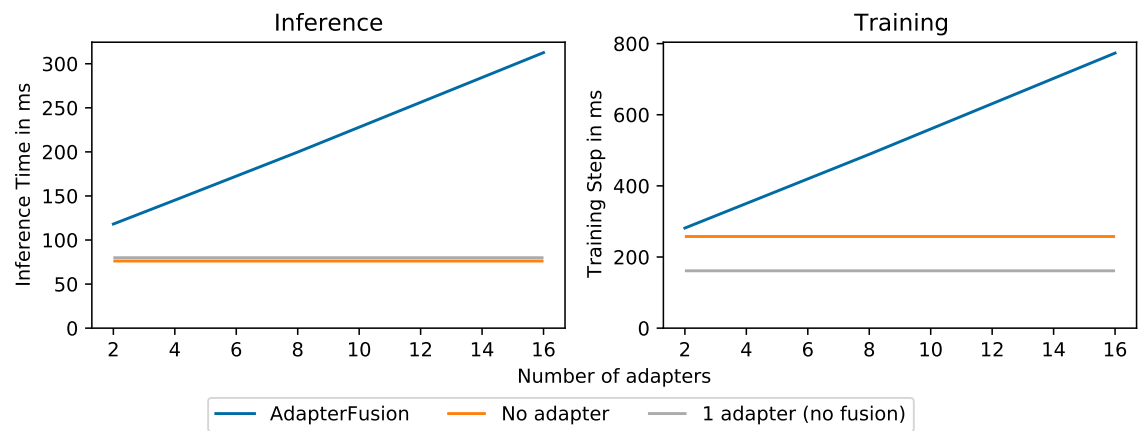

(a) V100

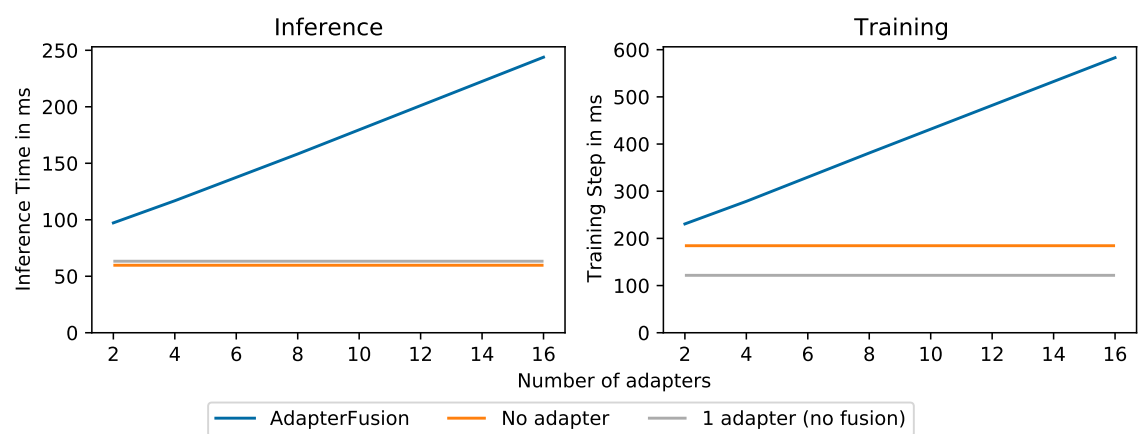

(b) TitanX

Figure 9: Absolute time measurements for AdapterFusion at inference (left) and training (right) as a function of the number of adapters. The measurements were conducted with a batch size of 32 (V100) and 16 (TitanX), and a sequence length of 128 . 


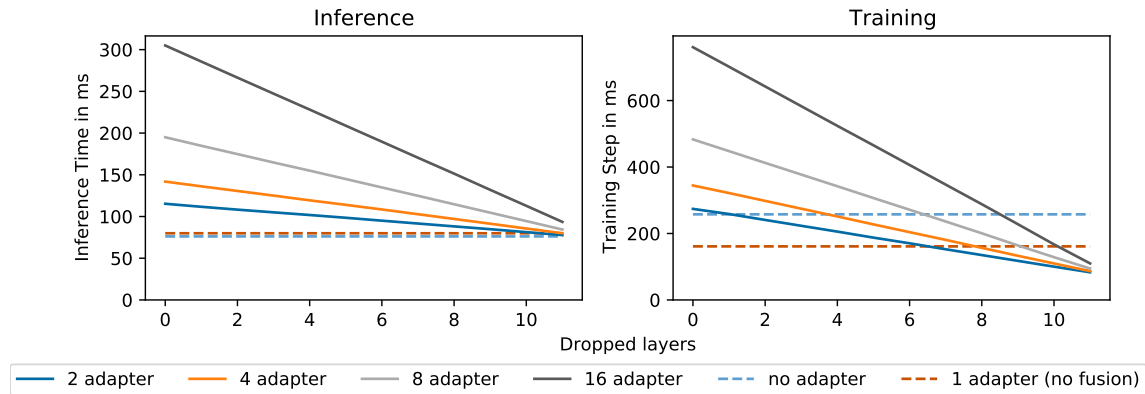

(a) V100

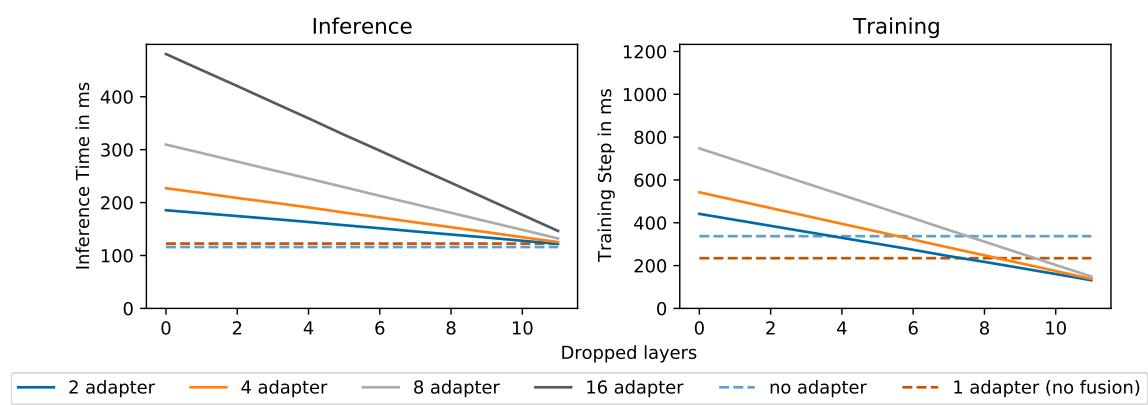

(b) $\operatorname{Titan} X$

Figure 10: Absolute time measurements for AdapterFusion with AdapterDrop at inference (left) and training (right) as a function of the number of dropped layers. The measurements were conducted with a batch size of 32 and a sequence length of 128 . We additionally plot the time of an adapter (without AdapterDrop) and a model without adapters to provide a more thorough comparison.

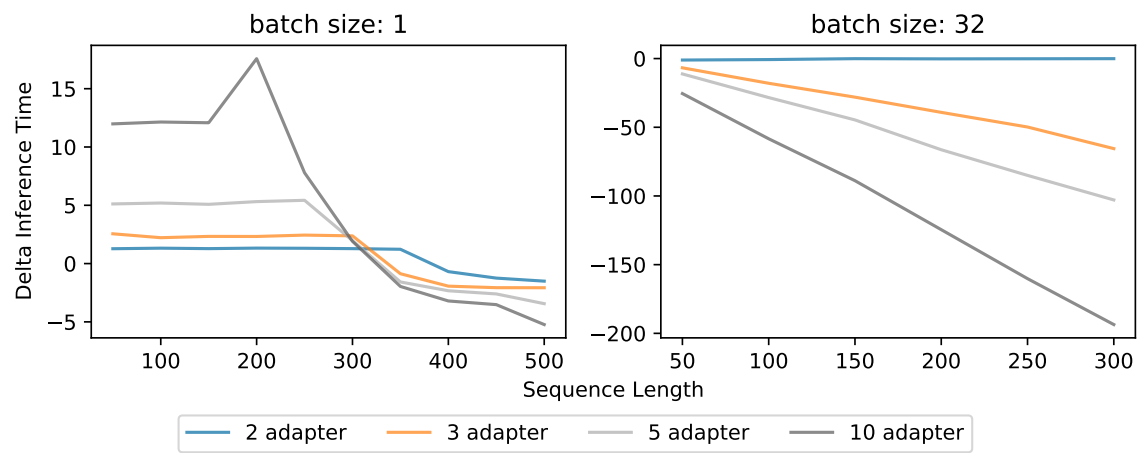

(a) V100

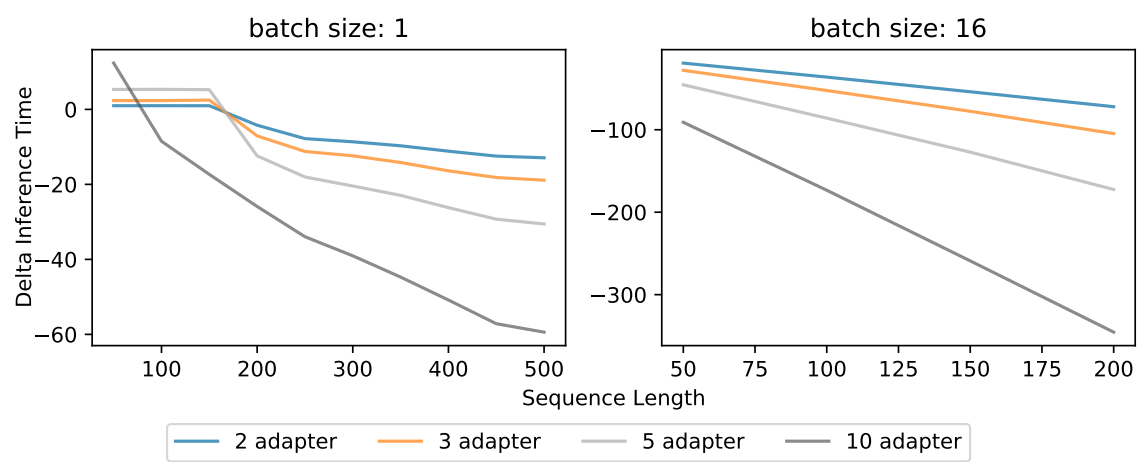

(b) $\operatorname{Titan} \mathrm{X}$

Figure 11: The difference in inference time between iterative and parallel implementations of AdapterFusion. Negative values indicate that the iterative implementation is faster. We calculate the difference as $t_{i}-t_{p}$, where $t_{i}, t_{p}$ are the times for iterative and parallel implementation, respectively. In Figure (a), the parallel implementation is faster if the input is sufficiently small as the GPU is not working at capacity and is able to use the parallel implementation. 

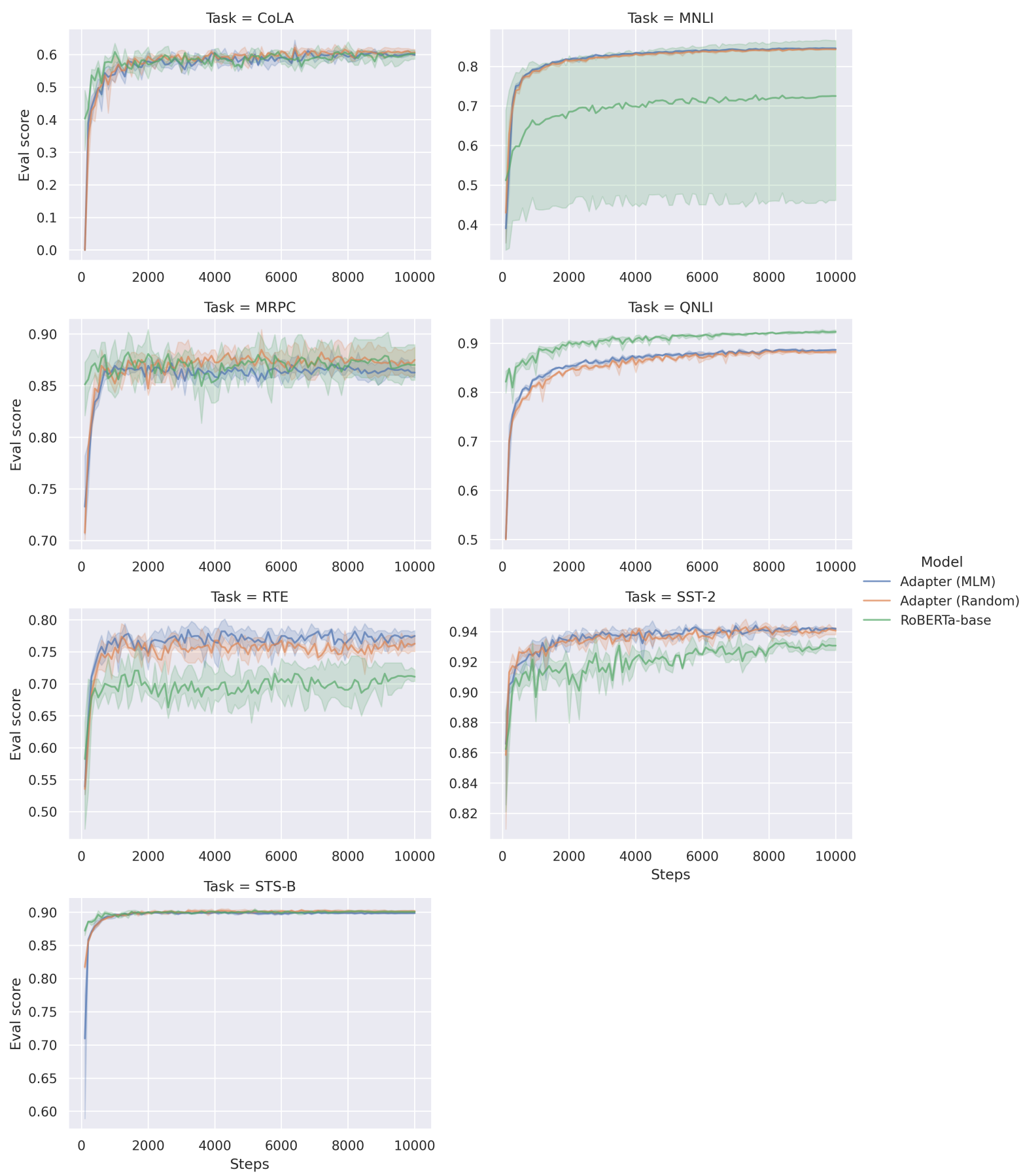

Figure 12: Evaluation performance of fine-tuning RoBERTA-base in comparison with different initialization strategies for adapters (randomly initialized vs. pre-trained on masked language modeling task). Training was conducted for 10k steps with a learning rate of 5e-05 for RoBERTa-base and 0.0001 for adapters, respectively. 

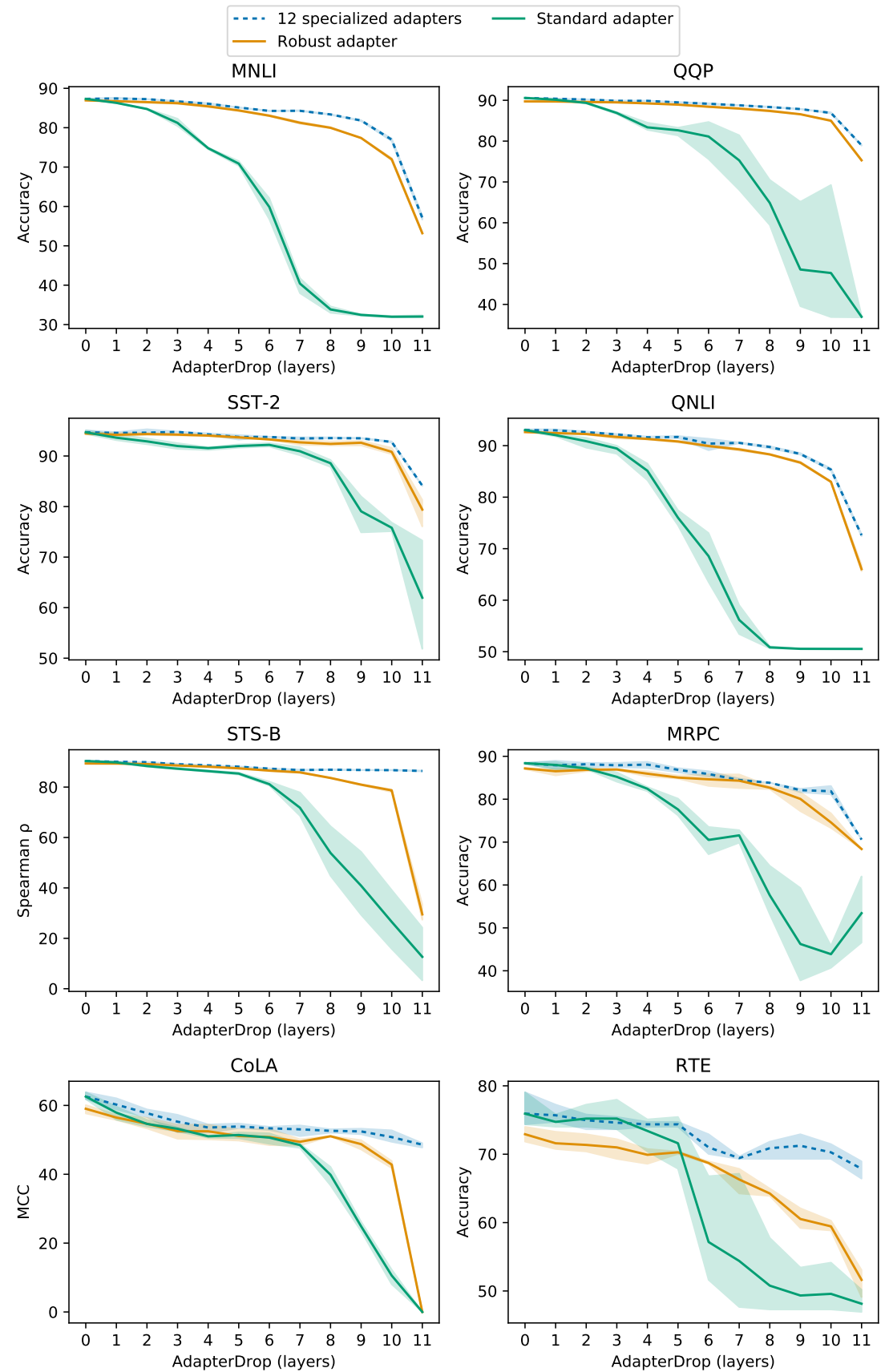

Figure 13: The AdapterDrop task performances for all eight GLUE tasks in relation to the dropped layers. ' 12 specialized adapters' refers to the performance of indiviudal models trained for each AdapterDrop setting separately (i.e., 12 models); 'Standard adapter' refers to the adapter that is trained with no dropped layers; AdapterDrop training refers to the adapter that is trained with our proposed training procedure. 